\title{
Dependent-Chance Goal Programming for Water Resources Management under Uncertainty
}

\author{
Haiying Guo, ${ }^{1}$ Honghua Shi, ${ }^{2}$ and Xiaosheng Wang ${ }^{1}$ \\ ${ }^{1}$ School of Science, Hebei University of Engineering, Handan 056038, China \\ ${ }^{2}$ School of Economics and Management, Hebei University of Engineering, Handan 056038, China \\ Correspondence should be addressed to Xiaosheng Wang; xswang@hebeu.edu.cn
}

Received 20 April 2016; Revised 4 July 2016; Accepted 25 July 2016

Academic Editor: Dan Ralescu

Copyright (C) 2016 Haiying Guo et al. This is an open access article distributed under the Creative Commons Attribution License, which permits unrestricted use, distribution, and reproduction in any medium, provided the original work is properly cited.

\begin{abstract}
Without sufficient data, consulting experts is a good way to quantify unknown parameters in water resources management which will result in human uncertainty. The aim of this paper is to introduce a new tool-uncertainty theory to deal with such uncertainty which is treated as uncertain variable with uncertainty distribution. And a dependent-chance goal programming (DCGP) model is provided for water resources management under such circumstance. In the model uncertain measure is used to measure possibility that an event will occur which is maximized by minimizing the deviation (positive or negative deviation) from target of objective event under a given priority structure. In the end, the developed model is applied to a numerical example to illustrate the effectiveness of the model. The result obtained contributes to the desired water-allocation schemes for decision-markers.
\end{abstract}

\section{Introduction}

The water resources management aims to enhance the effective use and guarantee sustainable development of water resources, when requirements about water quality and quantity of users are satisfied. Generally, there are several essential principles for water resources management: maximize the benefits in the development and utilization of water resources; emphasize joint operation of surface water and groundwater; lay equal stress on development and protection of water resources; strike a balance between water quality and water quantity. However, some natural and human factors (extreme climate events, emergency, population growth, etc.) bring severe challenges for efficient management of water resources, such as severe water shortage and water pollution. Obviously, these challenges will intensify the uncertainty of water resources management. However, in earlier study, researchers seldom consider uncertainty which already exists in water resources management. For example, Howes [1] applied linear programming to obtain doable water resource investments for water development projects. Revelle et al. [2] minimized the cost of the treatment plants in water quality management by using linear programming approach. Howard and Shamir [3] established a deterministic linear programming model to study interrelated land and water resource management problem. These approaches above did not take indeterminate factors into consideration.

However, in reality, uncertainty often influences decisionmaking in water resources allocation, utilization, scheduling, and protection. Later, researchers noted and treated uncertainty as randomness, fuzziness, or both of them. As a result, numerous mathematical methods have been proposed based on stochastic programming to tackle uncertainty treated as randomness. For example, Dupačová et al. [4] utilized stochastic programming to offer alternative decisions for decision-makers in water management. Feiring et al. [5] proposed a stochastic programming model for agricultural irrigation where rainfall was assumed to be a random variable. Huang and Loucks [6] proposed an inexact twostage stochastic programming model to deal with uncertainty where total water available and net benefit were unknown in water resources management. Kracman et al. [7] developed a multistage stochastic optimization model to handle uncertainty in the inflows into the Highland Lakes of the Highland Lakes system. Zhou et al. [8] proposed a factorial multistage stochastic programming approach for water resources management under uncertainty where some parameters of water allocation are indeterminate. Besides, some researchers used 
fuzzy programming to handle uncertainty presented as fuzzy set. For example, Jairaj and Vedula [9] formulated a fuzzy mathematical programming model based on fuzzy set theory for a multireservoir system. Sahoo et al. [10] used fuzzy optimization and liner programming to optimize the allocation of land and water resources. Zhang et al. [11] proposed an interactive inexact fuzzy bounded programming approach for agricultural water quality management. Sometimes, multiple uncertainties also exist in water resources management problems. Recent years, some methods are addressed through combining different mathematical programming method to deal multiple uncertainties. Guo et al. [12] developed a fuzzy stochastic two-stage programming approach for water resources management under randomness and fuzziness which integrated the fuzzy robust programming, chanceconstrained programming, and two-stage stochastic programming. Li et al. [13] proposed fuzzy-boundary intervalstochastic programming method to tackle uncertainties expressed as probability distributions and fuzzy-boundary intervals. Zeng et al. [14] developed a two-stage credibilityconstrained programming with Hurwicz criterion approach to tackle uncertainties presented as probability distributions and fuzzy sets.

These existing methods are effective to deal with objective uncertainty based on probability theory and fuzzy set theory. However, human uncertainty also exists in water resources management. Without sufficient data, consulting experts is another choice to quantify unknown parameter. In this case, human uncertainty always occurs. It is a fact that probability theory is a useful tool when a large number of sample data exists. So it is less effective to tackle such problems based on probability theory if there is lack of sample data. Besides, some researchers used fuzzy set theory to deal with human uncertainty. But it does not emphasize the law of excluded middle and the law of contradiction. In contrast, uncertainty theory [15] follows these principles which was founded in 2007 and became a branch of mathematics based on the normality, duality, and countable subadditivity. It is noteworthy that uncertainty theory is a new mathematical tool rather than a general term for all theory to handle uncertainty. Nowadays, it has been applied to different fields to tackle with uncertain phenomenon. For example, Liu proposed uncertain programming [16], uncertain multiobjective programming and uncertain goal programming [17], and uncertain multilevel programming [18] to deal with decision processing including belief degree successively.

Compared with probability theory and fuzzy set theory, uncertainty theory is effective in handling human uncertainty. So based on uncertainty theory, this paper considers the optimal allocation of water supply systems in uncertain environment. And uncertainty is expressed as uncertain variable with uncertainty distribution. Then the uncertain measure is used to measure possibility that an event will occur. The objective function of this model is to minimize the deviation from target of objective event under a given priority structure. Thus, DCDP model is formulated.

The rest of this paper is organized as follows. Section 2 introduces some basic concepts of uncertainty theory used throughout this paper. Section 3 presents a dependent-chance goal programming model for the water supply system under uncertain environment. Section 4 introduces algorithm to solve the model. Section 5 gives a numerical example to illustrate the effectiveness of the model. Finally, a conclusion is drawn in Section 6.

\section{Preliminaries}

In this section, we will introduce some useful definitions about uncertain measure, uncertain distribution, and so on.

Let $\Gamma$ be a nonempty set, and $\mathscr{L}$ be $\sigma$-algebra over $\Gamma$. Each element $\Lambda \in \mathscr{L}$ is called an event. A number $\mathscr{M}\{\Lambda\}$ indicates the possibility that $\Lambda$ will occur. Uncertain measure $\mathscr{M}$ is introduced as a set function satisfying the following axioms [15]:

Axiom 1 (normality axiom). $\mathscr{M}\{\Gamma\}=1$ for the universal set $\Gamma$.

Axiom 2 (duality axiom). $\mathscr{M}\{\Lambda\}+\mathscr{M}\left\{\Lambda^{c}\right\}=1$ for any event $\Lambda$.

Axiom 3 (subadditivity axiom). For every countable sequence of events $\left\{\Lambda_{i}\right\}$, we have

$$
\mathscr{M}\left\{\bigcup_{i=1}^{\infty} \Lambda_{i}\right\} \leq \sum_{i=1}^{\infty} \mathscr{M}\left\{\Lambda_{i}\right\} .
$$

The triplet $(\Gamma, \mathscr{L}, \mathscr{M})$ is called an uncertainty space. In addition, the product uncertain measure [19] was defined as follows.

Axiom 4 (product axiom). Let $\left(\Gamma_{k}, \mathscr{L}_{k}, \mathscr{M}_{k}\right)$ be uncertainty spaces for $k=1,2, \ldots$. The product uncertain measure $\mathscr{M}$ is an uncertain measure satisfying

$$
\mathscr{M}\left\{\prod_{k=1}^{\infty} \Lambda_{k}\right\}=\bigwedge_{k=1}^{\infty} \mathscr{M}_{k}\left\{\Lambda_{k}\right\} .
$$

The concept of uncertain variable $\xi$ was introduced [15] as a measurable function from an uncertainty space $(\Gamma, \mathscr{L}, \mathscr{M})$ to the set of real numbers. In order to describe an uncertain variable, uncertainty distribution is defined [15] as

$$
\Phi(x)=\mathscr{M}\{\xi \leq x\}, \quad \forall x \in \mathfrak{R} .
$$

Furthermore, the inverse uncertain distribution $\Phi^{-1}(\alpha)$ of $\xi$ was defined [20]. It plays a crucial role in operations of uncertain variable.

An uncertain variable $\xi$ is called normal if it has a normal uncertainty distribution [15]

$$
\Phi(x)=\left(1+\exp \left(\frac{\pi(e-x)}{\sqrt{3} \sigma}\right)\right)^{-1}, \quad x \in R,
$$

denoted by $N(e, \sigma)$, where $e$ and $\sigma$ are real numbers with $\sigma>$ 0 .

An uncertain variable $\xi$ is called lognormal if it has a normal uncertainty distribution [15]

$$
\Phi(x)=\left(1+\exp \left(\frac{\pi(e-\ln x)}{\sqrt{3} \sigma}\right)\right)^{-1}, \quad x \geq 0,
$$


denoted by $\operatorname{LOGN}(e, \sigma)$, where $e$ and $\sigma$ are real numbers with $\sigma>0$.

\section{DCGP Model under Uncertainty Environments}

Usually, optimal allocation of water supply system is a multiobjective programming problem. However, these goals are often contradictory and achievable at the cost of other goals. In this case, it is a preferred solution to create a priority structure. Then the lower-priority goals are considered after achieving the higher-priority goals. Under such circumstances, based on probability theory, dependent-chance goal programming (DCGP) [21] was firstly proposed to deal with the complex system which contains multiple resources and multiple users. Later the former one was improved and applicable for general stochastic decision systems [22], fuzzy decision systems [23], and fuzzy random systems [24]. Specifically, DCGP is a good tool to deal with water supply-allocation problem, production process [25], capital budgeting [26], and so on.

However, it is reasonable to accept the existence of the system (or phenomenon) which is neither random nor fuzzy. The phenomenon that there is lack of sufficient historical data is a case. In order to cope with such decision systems, uncertainty theory is used to formulate DCGP as follows:

$$
\min \sum_{j=1}^{l} P_{j} \sum_{i=1}^{m}\left(u_{i j} d_{i}^{+}+v_{i j} d_{i}^{-}\right)
$$

subject to: $M\left\{\begin{array}{c}h_{i k}(\mathbf{x}, \boldsymbol{\xi}) \leq 0 \\ k=1,2, \ldots, q_{i}\end{array}\right\}+d_{i}^{-}-d_{i}^{+}=b_{i}$,

$$
\begin{aligned}
& i=1,2, \ldots, m, \\
& g_{j}(\mathbf{x}, \boldsymbol{\xi}) \leq 0, \quad j=1,2, \ldots, p, \\
& d_{i}^{+}, d_{i}^{-} \geq 0, \quad i=1,2, \ldots, m,
\end{aligned}
$$

where some parameters are shown as follows: $P_{j}$ is the priority factor which represents the relative importance of each goal; $l$ is number of priorities; $u_{i j}$ is the weighting factor of positive deviation for goal $i$ under priority $j$ assigned; $v_{i j}$ is the weighting factor of negative deviation for goal $i$ under priority $j$ assigned; $m$ is the number of goal constraints; $b_{i}$ is given target value of goal $i$.

The objective event is expressed as $h_{i k}(\mathbf{x}, \boldsymbol{\xi}) \leq 0, \quad k=$ $1,2, \ldots, q_{i}, i=1,2, \ldots, m$, where $\mathbf{x}$ is decision vector and $\xi$ is uncertain vector. $M\left\{h_{i k}(\mathbf{x}, \boldsymbol{\xi}) \leq 0, k=1,2, \ldots, q_{i}, i=\right.$ $1,2, \ldots, m\}$ is uncertain measure of event occurrence. Let $f_{i}(\mathbf{x})$ be the chance function of the objective event

$$
f_{i}(\mathbf{x})=\left\{\begin{array}{c}
M\left\{h_{i k}(\mathbf{x}, \boldsymbol{\xi}) \leq 0\right\}, k=1,2, \ldots, q_{i} \\
g_{j}(\mathbf{x}, \boldsymbol{\xi}) \leq 0, j \in J_{i}
\end{array}\right\},
$$

where $J_{i}$ is the dependant constraints of objective event $i(i=$ $1,2, \ldots, m)$.

On the basis of chance functions and goal values, the deviations (positive or negative deviations) can be defined.

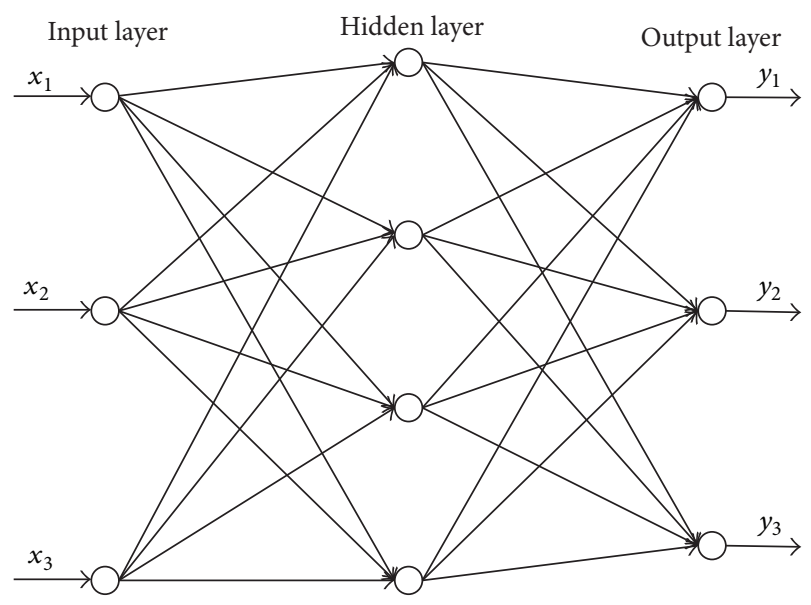

Figure 1: BP neural network structure.

Let $d_{i}^{+}$be the positive deviation from the target of goal $i$, denoted as

$$
d_{i}^{+}= \begin{cases}f_{i}(\mathbf{x})-b_{i}, & f_{i}(\mathbf{x})>b_{i}, \\ 0, & f_{i}(\mathbf{x}) \leq b_{i}, i=1,2, \ldots, m .\end{cases}
$$

Let $d_{i}^{-}$be the negative deviation from the target of goal $i$, denoted as

$$
d_{i}^{-}= \begin{cases}0, & f_{i}(\mathbf{x}) \geq b_{i}, \\ b_{i}-f_{i}(\mathbf{x}), & f_{i}(\mathbf{x})<b_{i}, i=1,2, \ldots, m .\end{cases}
$$

The constraints $g_{j}(\mathbf{x}, \boldsymbol{\xi}) \leq 0, j=1,2, \ldots, p$, are the uncertain environment.

\section{Hybrid Intelligent Algorithm}

The hybrid intelligent algorithm which integrates uncertain simulation, neural network, and genetic algorithm is introduced to solve the model. The algorithm combines the global searching ability of genetic algorithm and the good local searching ability of neural network and improves training effect of neural network by using uncertain simulation.

To be more specific, the aim of uncertain simulation is to generate large amounts of input-output data. And neural network attempts to seek suitable weighting vector to approximate the uncertain functions by training inputoutput data (general network structure is shown in Figure 1). Finally, the optimum solution is obtained by applying genetic algorithm.

In conclusion, the concrete step of algorithm is just as follows (the framework of hybrid intelligent algorithm is shown in Figure 2).

Step 1. Apply uncertain simulation to generate input-output data for following uncertain functions; the number of simulations is $T$ every time through the loop:

$$
\begin{aligned}
U & : x \longrightarrow M\left\{h_{k}(\mathbf{x}, \boldsymbol{\xi}) \leq 0, k=1,2, \ldots, q ; g_{j}(\mathbf{x}, \boldsymbol{\xi})\right. \\
& \leq 0, j \in J\} .
\end{aligned}
$$




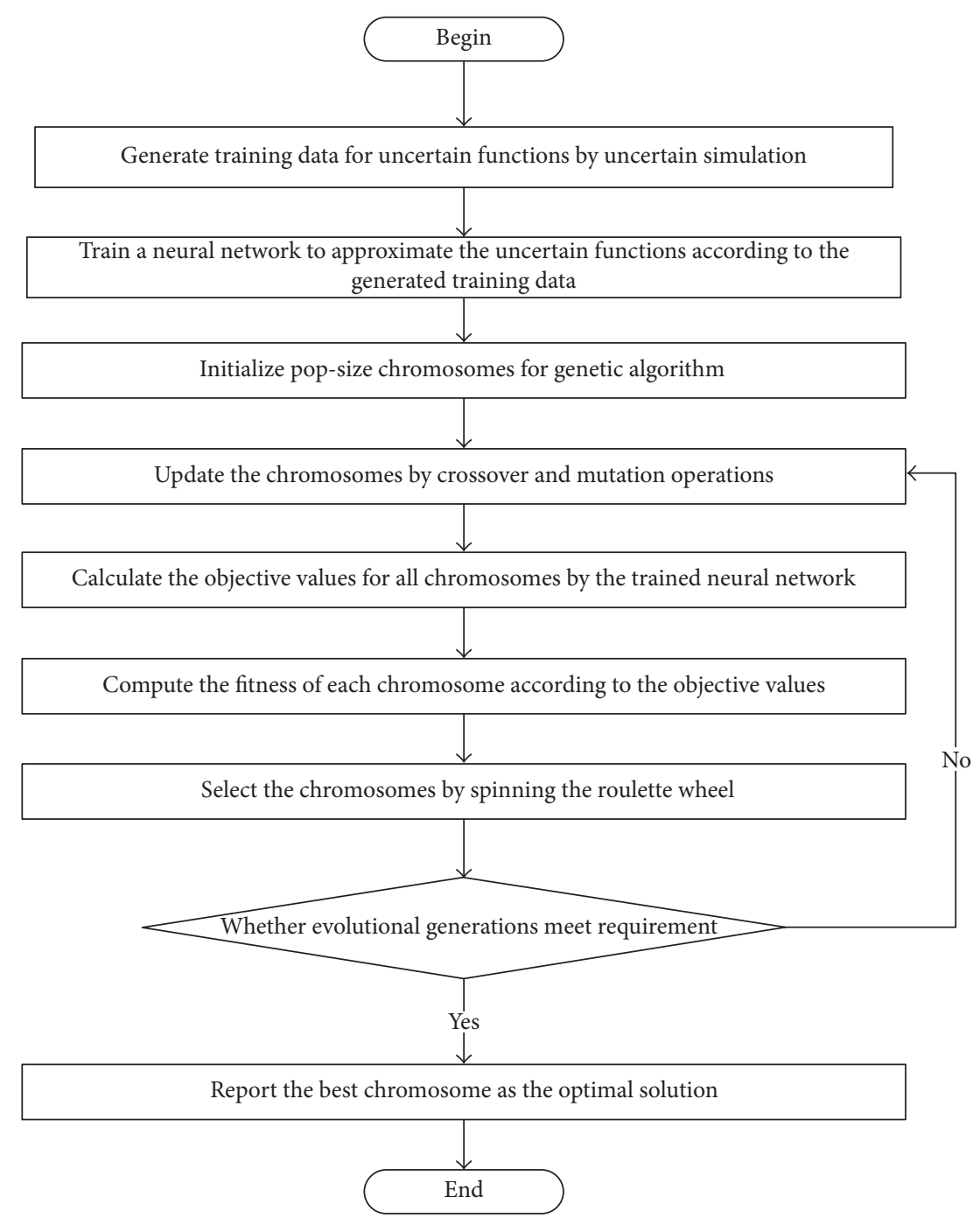

FIGURE 2: Framework of hybrid intelligent algorithm.

Step 2. Train a neural network according to input-output data generated by uncertain simulation. The error between training output and training data is minimized to approximate the uncertain functions. Finish train until training results are satisfying.

Step 3. Generate initial pop-size chromosomes randomly and check the feasibility of the chromosomes.

Step 4. Update the chromosomes by crossover and mutation operations.

Step 5. Calculate the objective values for all chromosomes by the trained neural network.

Step 6. Calculate the fitness of each chromosome in accordance with the objectives values by evaluation function.

Step 7. Choose the chromosomes by spinning the roulette wheel to create new chromosomes.
Step 8. Repeat Steps 4-7 until the number of cycles required.

Step 9. Select the best chromosome as the optimal solution of the model.

\section{Application in Water Resources Management}

In this section, let us consider a water supply and allocation problem shown in Figure 3. There are three newly constructed reservoirs for emergency water supply and three water users which are urban domestic, industrial, and agricultural sectors. In order to realize the demand of users, several problems need to be solved. Firstly, we need to consider the water quantity supplied by each reservoir. Secondly, the decisions to optimize the allocation of water resources should be made.

In order to solve above problems, we introduce 9 decision variables. $x_{1}, x_{2}$, and $x_{3}$ are quantities supplied from reservoir 1 to user $1,2,3$, respectively; $x_{4}, x_{5}$, and $x_{6}$ are quantities 


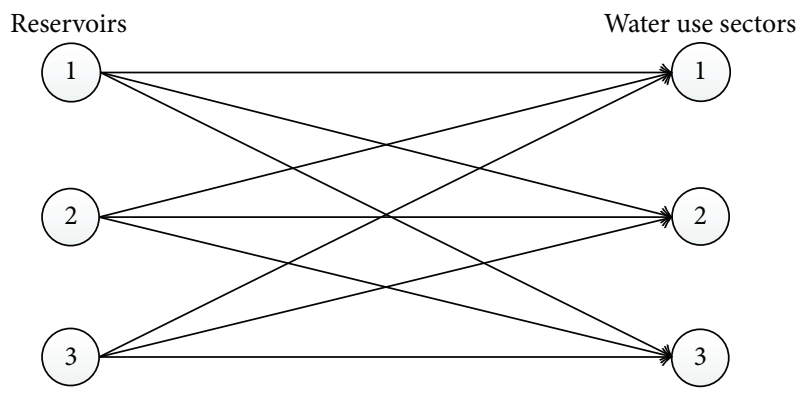

FIGURE 3: Water supply system.

supplied from reservoir 2 to user $1,2,3$, respectively; $x_{7}, x_{8}$, and $x_{9}$ are quantities supplied from reservoir 3 to user $1,2,3$, respectively. Remarkably, the water quantity supplied by each reservoir cannot exceed its maximum storage capacity which are marked by $\xi_{1}, \xi_{2}, \xi_{3}$. However, it is a challenge to quantify the maximum quantity without data about newly constructed reservoir. So let $\xi_{1}, \xi_{2}, \xi_{3}$ be independent uncertain variables with uncertainty distributions $\Phi_{1}, \Phi_{2}, \Phi_{3}$, respectively. Then the constraint are as follows:

$$
\begin{aligned}
& x_{1}+x_{2}+x_{3} \leq \xi_{1}, \\
& x_{4}+x_{5}+x_{6} \leq \xi_{2}, \\
& x_{7}+x_{8}+x_{9} \leq \xi_{3} .
\end{aligned}
$$

At the same time, the demands of three users must be satisfied, marked by $c_{1}, c_{2}, c_{3}$. Then three are three events

$$
\begin{aligned}
& x_{1}+x_{4}+x_{7}=c_{1} \\
& x_{2}+x_{5}+x_{8}=c_{2} \\
& x_{3}+x_{6}+x_{9}=c_{3} .
\end{aligned}
$$

Since emergencies happen, three newly constructed reservoirs are adoptable for emergency water supply and may not satisfied for all users. In this case, priority structure of three water sectors is supposed as follows.
Priority 1. The water supply rate in urban domestic reaches the given goal $b_{1}$ as much as possible.

Priority 2. The water supply rate in industry reaches the given goal $b_{2}$ as much as possible.

Priority 3. The water supply rate in agriculture reaches the given goal $b_{3}$ as much as possible.

Then the DCGP model is formulated:

$$
\begin{aligned}
\text { lexmin } & \left\{d_{1}^{-}, d_{2}^{-}, d_{3}^{-}\right\} \\
\text {subject to: } & M\left\{x_{1}+x_{4}+x_{7}=c_{1}\right\}+d_{1}^{-}-d_{1}^{+}=b_{1} \\
& M\left\{x_{2}+x_{5}+x_{8}=c_{2}\right\}+d_{2}^{-}-d_{2}^{+}=b_{2} \\
& M\left\{x_{3}+x_{6}+x_{9}=c_{3}\right\}+d_{3}^{-}-d_{3}^{+}=b_{3} \\
& x_{1}+x_{2}+x_{3} \leq \xi_{1} \\
& x_{4}+x_{5}+x_{6} \leq \xi_{2} \\
& x_{7}+x_{8}+x_{9} \leq \xi_{3} \\
& x_{i} \geq 0, \quad i=1,2, \ldots, 9 \\
& d_{i}^{-}, d_{i}^{+} \geq 0, \quad i=1,2,3 .
\end{aligned}
$$

Now, suppose that the maximum quantities $\xi_{1}, \xi_{2}, \xi_{3}$ are uncertain variables with uncertainty distributions $\operatorname{LOGN}(2.2,0.5), \operatorname{LOGN}(1.1,0.1)$, and $\operatorname{LOGN}(1.2,0.3)$, respectively. The water demands of three users $c_{1}, c_{2}$, and $c_{3}$ are 2,3 , and 1 , respectively. And the given goals $b_{1}, b_{2}$, and $b_{3}$ are $0.95,0.90$, and 0.85 , respectively.

The hybrid intelligent algorithm is used to solve this model. And the values of each parameter are shown in Table 1.

A run of the hybrid intelligent algorithm shows that the optimal solution is

$$
x=(1.0805,1.4490,0.1755,0.1871,0.5351,0.4300,0.7324,1.0159,0.3945)
$$

So, the optimal scheme is

quantities supplied from reservoir 1 to user 1,2, 3 are 1.0805, 1.4490, 0.1755;

quantities supplied from reservoir 2 to user 1, 2, 3 are $0.1871,0.5351,0.4300$;

quantities supplied from reservoir 3 to user 1, 2, 3 are $0.7324,1.0159,0.3945$.

The result shows that quantities supplied by three newly constructed reservoirs meet the demand of users. So, decision-makers can set higher goals and make water supply plans according to obtained result.

In reality, multiple objectives are usually in conflict and can not be achieved simultaneously in water supplyallocation problem. In order to realize the global optimization, it is an alternative approach for decision-makers to create a priority structure and set values for these goals according to their contribution to decision-making. Thus it not only gives consideration to all goals at the same time but also assures the achievement of the higher-priority goals as much as possible. 
TABLE 1: Value of each parameter.

\begin{tabular}{lcc}
\hline Number of loops & $T$ & 6000 \\
Input-output data & $D$ & 3000 \\
Number of input neurons & $N$ & 6 \\
Number of hidden neurons & $H$ & 15 \\
Number of output neurons & $O$ & 3 \\
Population size & Pop-size & 30 \\
Parameter of evaluation function & $a$ & 0.5 \\
Crossover probability & $P_{c}$ & 0.3 \\
Mutation probability & $P_{m}$ & 0.2 \\
Number of iterations & Gen & 1000 \\
\hline
\end{tabular}

\section{Conclusions}

This study proposes a dependent-chance goal programming model under uncertain environment in the absence of historical data as reference. Different from previous dependentchance goal programming under random and environment, the uncertain variable and uncertain measure are brought into the model based on uncertainty theory. In the model, possibility of an event's occurrence is measured by uncertain measure. The objective is to maximize the chance of an event's occurrence by means of minimizing the deviation (positive or negative deviation) from target of objective event under a given priority structure. Finally, an example is successfully solved to demonstrate the validity of the proposed model.

\section{Competing Interests}

The authors declare that there are no competing interests regarding the publication of this paper.

\section{Acknowledgments}

This work was supported by Hebei Natural Science Foundations (no. G2013402063).

\section{References}

[1] R. Howes, "A test of a linear programming model of agriculture," Papers of the Regional Science Association, vol. 19, no. 1, pp. 123140, 1967.

[2] C. S. Revelle, D. P. Loucks, and W. R. Lynn, "Linear programming applied to water quality management," Water Resources Research, vol. 4, no. 1, pp. 1-9, 1968.

[3] C. D. D. Howard and U. Shamir, "Hierarchical multiple objective analysis of land and water resource utilization for a system of lakes," in Large Engineering Systems, pp. 596-606, 1977.

[4] J. Dupačová, A. Gaivoronski, Z. Kos, and T. Szántai, "Stochastic programming in water management: a case study and a comparison of solution techniques," European Journal of Operational Research, vol. 52, no. 1, pp. 28-44, 1991.

[5] B. R. Feiring, T. Sastri, and L. S. Sim, "A Stochastic programming model for water resource planning," Mathematical and Computer Modelling, vol. 27, no. 3, pp. 1-7, 1998.
[6] G. H. Huang and D. P. Loucks, "An inexact two-stage stochastic programming model for water resources management under uncertainty," Civil Engineering and Environmental Systems, vol. 17, no. 2, pp. 95-118, 2000.

[7] D. R. Kracman, D. C. McKinney, D. W. Watkins Jr., and L. S. Lasdon, "Stochastic optimization of the Highland Lakes system in Texas," Journal of Water Resources Planning and Management, vol. 132, no. 2, pp. 62-70, 2006.

[8] Y. Zhou, G. H. Huang, and B. Yang, "Water resources management under multi-parameter interactions: a factorial multistage stochastic programming approach," Omega, vol. 41, no. 3, pp. 559-573, 2012.

[9] P. G. Jairaj and S. Vedula, "Multireservoir system optimization using fuzzy mathematical programming," Water Resources Management, vol. 14, no. 6, pp. 457-472, 2000.

[10] B. Sahoo, A. K. Lohani, and R. K. Sahu, "Fuzzy multiobjective and linear programming based management models for optimal land-water-crop system planning," Water Resources Management, vol. 20, no. 6, pp. 931-948, 2006.

[11] Y. M. Zhang, H. W. Lu, X. H. Nie, L. He, and P. Du, "An interactive inexact fuzzy bounded programming approach for agricultural water quality management," Agricultural Water Management, vol. 133, pp. 104-111, 2014.

[12] P. Guo, G. H. Huang, H. Zhu, and X. L. Wang, "A twostage programming approach for water resources management under randomness and fuzziness," Environmental Modelling \& Software, vol. 25, no. 12, pp. 1573-1581, 2010.

[13] Y. P. Li, G. H. Huang, and S. L. Nie, "Planning water resources management systems using a fuzzy-boundary intervalstochastic programming method," Advances in Water Resources, vol. 33, no. 9, pp. 1105-1117, 2010.

[14] X. T. Zeng, Y. P. Li, W. Huang, X. Chen, and A. M. Bao, “Twostage credibility-constrained programming with Hurwicz criterion (TCP-CH) for planning water resources management," Engineering Applications of Artificial Intelligence, vol. 35, pp. 164-175, 2014.

[15] B. D. Liu, Uncertainty Theory, Springer, Berlin, Germany, 2nd edition, 2007.

[16] B. D. Liu, Theory and Practice of Uncertain Programming, Springer, Berlin, Germany, 2nd edition, 2009.

[17] B. D. Liu and X. W. Chen, "Uncertain multiobjective programming and uncertain goal programming," Tech. Rep., 2012.

[18] B. D. Liu and K. Yao, "Uncertain multilevel programming: algorithm and applications," Computers \& Industrial Engineering, vol. 89, pp. 235-240, 2015.

[19] B. D. Liu, "Some research problems in uncertainty theory", Journal of Uncertain Systems, vol. 3, no. 1, pp. 3-10, 2009.

[20] B. D. Liu, "Why is there a need for uncertainty theory?" Journal of Uncertain Systems, vol. 6, no. 1, pp. 3-10, 2012.

[21] B. D. Liu and J. F. Gu, "Dependent-chance goal programming and an application," Journal of Systmes Engineering \& Electronics, vol. 4, no. 2, pp. 40-47, 1993.

[22] B. D. Liu, "Dependent-chance goal programming and its genetic algorithm based approach," Mathematical and Computer Modelling, vol. 24, no. 7, pp. 43-52, 1996.

[23] B. D. Liu, "Dependent-chance programming with fuzzy decisions," IEEE Transactions on Fuzzy Systems, vol. 7, no. 3, pp. 354360, 1999. 
[24] B. D. Liu, "Fuzzy random dependent-chance programming," IEEE Transactions on Fuzzy Systems, vol. 9, no. 5, pp. 721-726, 2001.

[25] B. D. Liu and K. Iwamura, "Modelling stochastic decision systems using dependent-chance programming," European Journal of Operational Research, vol. 101, no. 1, pp. 193-203, 1997.

[26] R. Liang and J. W. Gao, "Dependent-chance programming models for capital budgeting in fuzzy environments," Tsinghua Science and Technology, vol. 13, no. 1, pp. 117-120, 2008. 

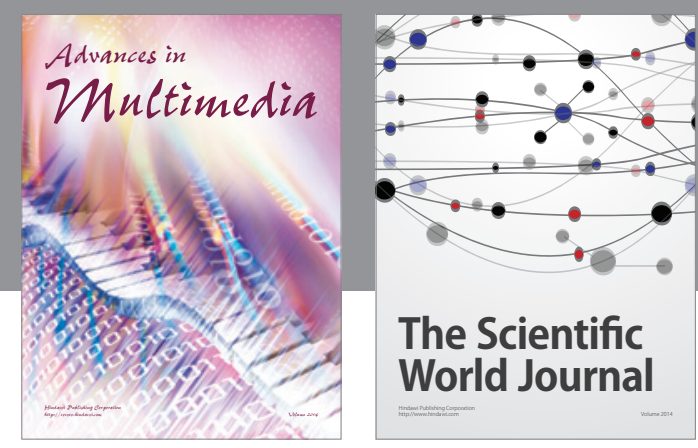

The Scientific World Journal
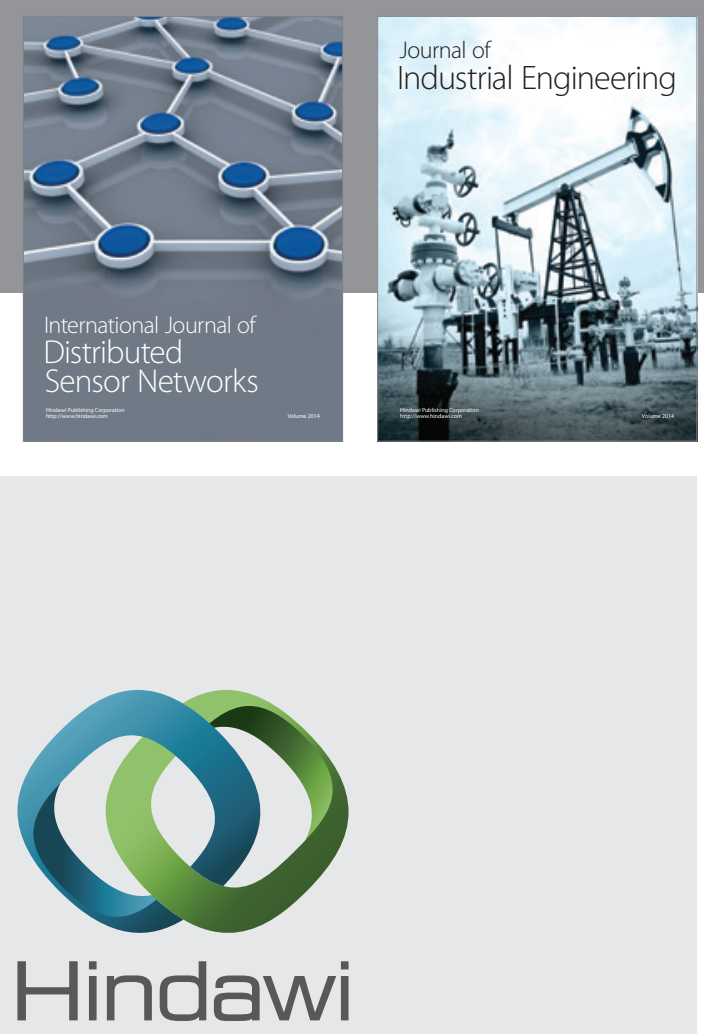

Submit your manuscripts at

http://www.hindawi.com

\section{Computer Networks} and Communications
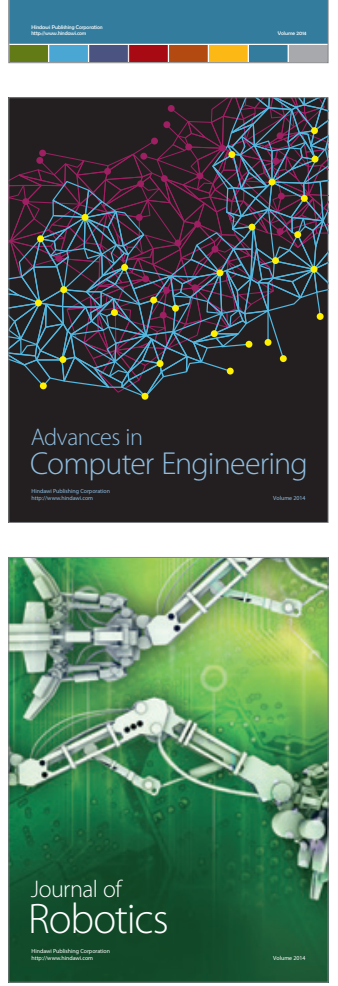
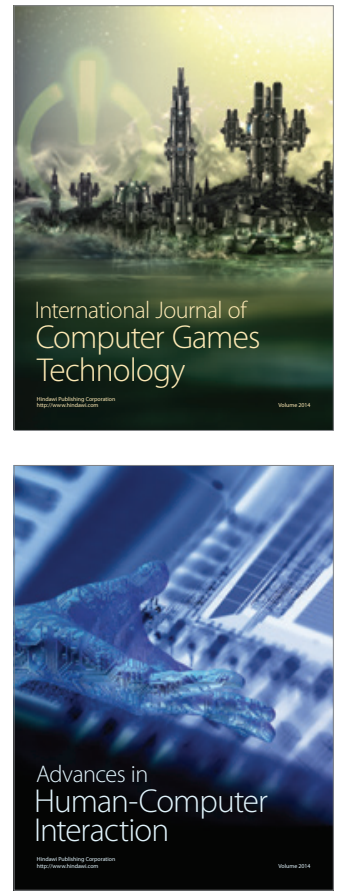
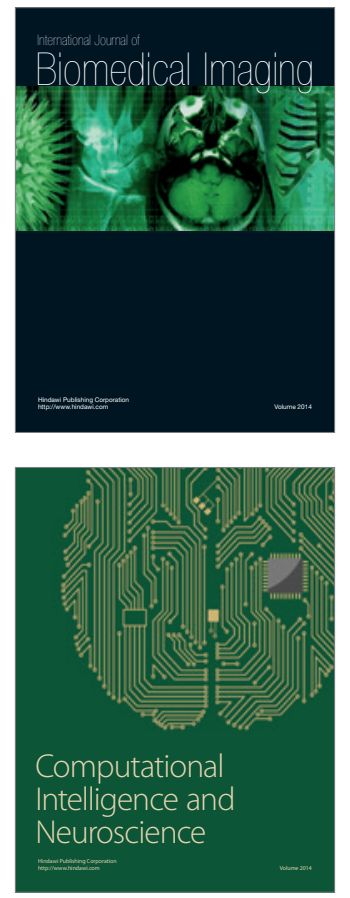
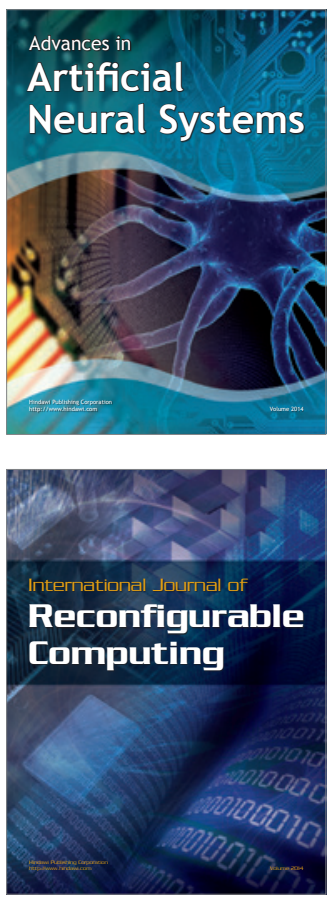
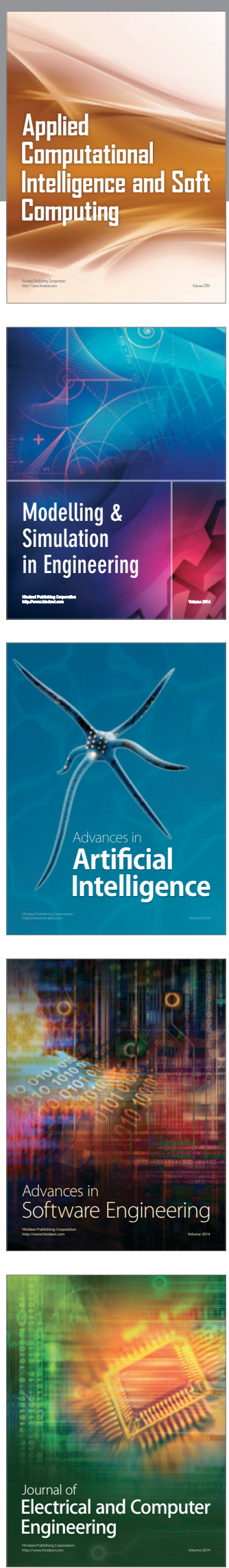